See Article page 1022.

\section{Commentary: Minimally invasive aortic valve replacement in the transcatheter aortic valve replacement era: Where do we stand?}

\author{
Charles Laurin, MD, and François Dagenais, MD
}

Bonacchi and colleagues ${ }^{1}$ report a study comparing right minithoracotomy (RT) and ministernotomy (MS) for minimally invasive aortic valve replacement (AVR). Through propensity score matching of 2419 patients operated over a 20-year period, 986 patients were matched in each group. During the same period, 3175 patients underwent operation for an isolated AVR through a standard sternotomy approach; no control sternotomy group was included in the study. The authors reported increased perioperative mortality, intensive care unit stay, and hospitalization in the minithoracotomy (MT) group. A conversion rate of $27.7 \%$ was observed in the MT group; the rate reduced to $15.4 \%$ following implementation of preoperative computed tomography (CT) planning in 2009. MT remained a significant predictor of poor long-term survival at a median follow-up of 10 years.

During the past 2 decades, in an effort to lessen perioperative morbidity, innovations in cardiac surgery led to the development of less-invasive procedures, including MT and RT for AVR procedures. Different centers reported excellent outcomes with minimally invasive AVR procedures; either with MT or MS. ${ }^{2}$ However, no randomized trial compares outcomes of the 2 minimally

\footnotetext{
From the Department of Cardiac Surgery, Institut Universitaire de Cardiologie et de Pneumologie de Québec, Université Laval, Québec, Québec, Canada.

Disclosures: The authors reported no conflicts of interest.

The Journal policy requires editors and reviewers to disclose conflicts of interest and to decline handling or reviewing manuscripts for which they may have a conflict of interest. The editors and reviewers of this article have no conflicts of interest.

Received for publication May 9, 2021; revisions received May 9, 2021; accepted for publication May 10, 2021; available ahead of print May 14, 2021.

Address for reprints: François Dagenais, MD, Department of Cardiac Surgery, Institut Universitaire de Cardiologie et de Pneumologie de Québec, 2725 Chemin SainteFoy, Québec, Québec G1V 4G5 Canada (E-mail: francois.dagenais@chg.ulaval. ca).

J Thorac Cardiovasc Surg 2023;165:1033-4

$0022-5223 / \$ 36.00$

Copyright (c) 2021 by The American Association for Thoracic Surgery

https://doi.org/10.1016/j.jtcvs.2021.05.020
}

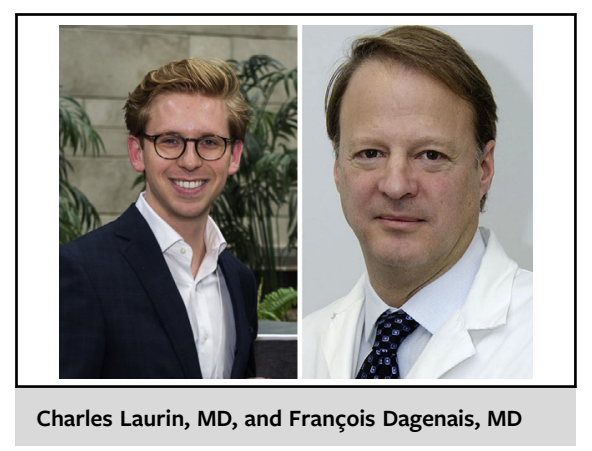

CENTRAL MESSAGE

Minimally invasive AVR is an important tool in the armamentarium of the cardiac surgeon and requires preoperative planning and surgical expertise to ensure optimal perioperative outcomes.

invasive AVR approaches or to a standard full sternotomy approach. The present trial has the merit of matching a large cohort of MT/RT patients undergoing operation in 5 centers; hence, better reflecting real-world outcomes. Although sutureless valves have been suggested to facilitate the RT approach, ${ }^{3}$ the present trial implanted all types of AVR substitutes in a similar ratio for both approaches. The high conversion rate is concerning and supports the importance of strict CT criteria for patient selection and operative planning as well as surgical expertise. Although not stated, the timing of the conversion may have contributed to the higher mortality in the RT group. In addition to the surgical conversion rate, the greater surgical complexity-hence learning curve-of the RT procedure is supported by an increase in operative and cardiopulmonary bypass times. Although perioperative morbidity was increased in the RT group, significant limitations should be addressed. The RT approach was performed mainly before 2009, whereas the MS was the preferred technique thereafter. Implementation of early extubation and fast-track protocols during the past decade may potentially bias data such as extubation time, intensive care unit stay, and hospital stay in favor of the MS group. However, because a groin cannulation is required more often with an RT approach, a higher rate of groin complications is inherent with the RT 
approach and should be considered in patient selection. Similarly, as demonstrated by the authors, pleural complications are expected to be higher with the RT approach.

The transcatheter aortic valve replacement (TAVR) revolution decreased significantly the rates of open isolated AVR procedures. In most centers, open AVR is currently performed in young, mainly bicuspid patients, combined with other cardiac procedures or in patients not suitable for TAVR. Isolated AVR patients amenable to minimally invasive AVR procedures will be less-often encountered, although failed TAVR patients will generate a new clientele with specific surgical challenges, especially for minimally invasive surgical approaches. Although new instrumentation such as automated knot-tying devices may facilitate $\mathrm{RT}$ procedures, the learning curve is steeper than with MS and mandates careful preoperative planning and surgical expertise to ensure acceptable clinical outcomes. Randomized trials comparing minimally invasive AVR approaches to standard sternotomy are essential to assess the advantages and limitations of each technique. Bonacchi and colleagues ${ }^{1}$ have to be congratulated for their contribution to the evolving field of AVR.

\section{References}

1. Bonacchi M, Dokollari A, Parise O, Sani G, Prifti E, Bisleri G, et al. Ministernotomy compared with right anterior minithoracotomy for aortic valve surgery. J Thorac Cardiovasc Surg. 2023;165:1022-32.e2.

2. Balmforth D, Harky A, Lall K, Uppal R. Is ministernotomy superior to right anterior minithoracotomy in minimally invasive aortic valve replacement. Interac Cardiovasc Thorac Surg. 2017;25:818-21.

3. Glauber M, Ferrarini M, Miceli A. Minimally invasive aortic valve surgery: state of the art and future directions. Ann Cardiothorac Surg. $2015 ; 4: 26-32$
See Article page 1022.

\section{Commentary: Is minimally invasive cardiac surgery a Chimera?}

\author{
Vicente Orozco-Sevilla, MD, ${ }^{\mathrm{a}, \mathrm{b}, \mathrm{c}}$ and \\ Tomas A. Salerno, MD $^{\mathrm{d}}$
}

Minimally invasive aortic valve replacement (AVR) is gaining in popularity among patients, surgeons, and referring physicians. It is unclear whether mini-sternotomy (MS) or right anterior mini-thoracotomy (MT) results in less pain, fewer transfusions, shorter hospital stays with faster

\footnotetext{
From the a Division of Cardiothoracic Surgery, Michael E. DeBakey Department of Surgery, Baylor College of Medicine, Houston, Tex; ${ }^{\mathrm{b}}$ Section of Adult Cardiac Surgery, Department of Cardiovascular Surgery, Texas Heart Institute, Houston, Tex;

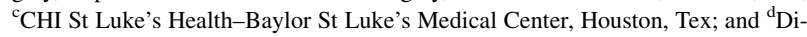
vision of Cardiothoracic Surgery, Miller School of Medicine, University of Miami, and Jackson Memorial Hospital, Miami, Fla.

Disclosures: The authors reported no conflicts of interest.

The Journal policy requires editors and reviewers to disclose conflicts of interest and to decline handling or reviewing manuscripts for which they may have a conflict of interest. The editors and reviewers of this article have no conflicts of interest.

Received for publication April 22, 2021; revisions received April 22, 2021; accepted for publication April 23, 2021; available ahead of print April 30, 2021

Address for reprints: Tomas A. Salerno, MD, Division of Cardiothoracic Surgery, University of Miami School of Medicine (R-114), 1611 NW 12th Ave, Miami, FL 33136 (E-mail: tsalerno@med.miami.edu).

J Thorac Cardiovasc Surg 2023;165:1034-5

$0022-5223 / \$ 36.00$

Copyright (c) 2021 by The American Association for Thoracic Surgery

https://doi.org/10.1016/j.jtcvs.2021.04.070
}

Check for updates

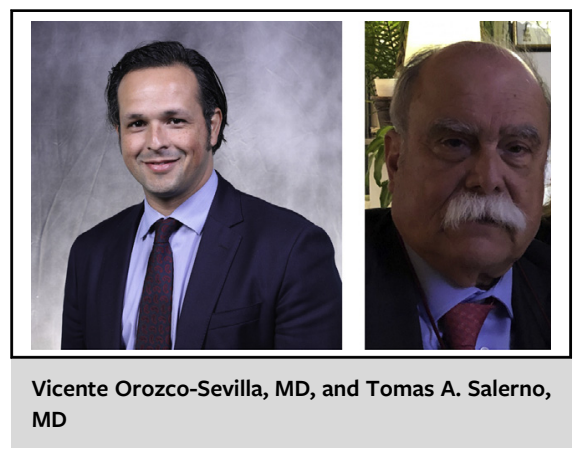

CENTRAL MESSAGE

Although mini-sternotomy and right anterior mini-thoracotomy

are gaining popularity, conven-

tional median sternotomy re-

mains the gold standard for any

AVR procedure.

recoveries, and even lower 30-day mortality, when compared with conventional median sternotomy. ${ }^{1,2}$ There are currently no guidelines or recommendations to guide the surgeon in determining which approach/incision to use, ie, MS, MT, or conventional median sternotomy. The majority of cardiac surgeons and trainees have no training in, or exposure to, MS or MT. This procedure is not difficult, but it does necessitate the acquisition of new and 